\title{
Effect of Ionic Liquids on the Separation of Sucrose Crystals from a Natural Product Using Crystallization Techniques
}

\author{
Ji-Hun An ${ }^{1,+}$ (D), Alice Nguvoko Kiyonga ${ }^{1,+}$, Woojin Yoon ${ }^{2}$, Minho Park ${ }^{1}$, Changjin Lim ${ }^{1}$, \\ Younghwi Yun ${ }^{1}$, Gyu-Hwan Park ${ }^{3, *}$ and Kiwon Jung ${ }^{1, *}$ \\ 1 College of Pharmacy, CHA University, Sungnam 13844, Korea; ajh@chauniv.ac.kr (J.-H.A.); \\ gabriella@chauniv.ac.kr (A.N.K.); minho.park92@gmail.com (M.P.); koryoi0709@gmail.com (C.L.); \\ jenniferyh@naver.com (Y.Y.) \\ 2 Department of Chemistry and Energy Systems Research, Ajou University, Suwon 16499, Korea; \\ xtal@ajou.ac.kr \\ 3 College of Pharmacy, Kyungpook National University, Daegu 41566, Korea \\ * Correspondence: park014@knu.ac.kr (G.-H.P.); pharmj@cha.ac.kr (K.J.); \\ Tel.: +82-53-9508576 (G.-H.P.); +82-31-8817173 (K.J.) \\ + These authors contributed equally to this work.
}

Academic Editor: Helmut Cölfen

Received: 26 August 2017; Accepted: 20 September 2017; Published: 23 September 2017

\begin{abstract}
The present work aims to investigate the applicability of ionic liquids (ILs) for natural ingredient crystallization. First, the medicinal plant, namely Angelica gigas Nakai, was extracted using methanol $(\mathrm{MeOH})$ as a solvent. Afterwards, ILs 1-butyl-3-methylimidazolium tetrafluoroborate $\left(\mathrm{BMImBF}_{4}\right)$, 1-butyl-3-methylimidazolium hexafluorophosphate $\left(\mathrm{BMImPF}_{6}\right)$, 1-butyl-3-methylimidazolium bis(trifluoromethylsulfonyl)imide (BMImTFSI), 1-allyl-3-ethylim idazolium tetrafluoroborate $\left(\mathrm{AEImBF}_{4}\right)$, and 1,3-diallyl imidazolium tetrafluoroborate $\left(\mathrm{AAImBF}_{4}\right)$, in three ratios of 1:1,1:2, and 1:3 (extraction solution/ILs $(v / v)$ ) were used as an anti-solvent to induce crystallization. Crystals were obtained within $8 \mathrm{~h}$ and were then identified to be pure crystals of sucrose through nuclear magnetic resonance $\left({ }^{1} \mathrm{H}-\mathrm{NMR}\right)$ analysis. Moreover, the single-crystal X-ray diffraction (SXD) analysis revealed all recovered crystals have an identical crystal structure and the morphology was monitored using a video microscope. With the application of BMImBF 4 and $\mathrm{BMImPF}_{6}$, transformation of sucrose crystal morphology from an elongated hexagon shape to an elongated rectangular shape was observed with respect to the respective concentration increase. Here, all crystals precipitated from $\mathrm{BMImBF}_{4}$ and $\mathrm{BMImPF}_{6}$ were found to possess identical PXRD patterns. However, when BMImTFSI was employed, small rectangular crystals attached to the larger rectangular-shaped crystals due to secondary nucleation and shapeless amorphous forms were observed according to the alteration in the solution to ILs ratio. Accordingly, the ability of ILs as a relevant anti-solvent for the selective crystallization of a single compound from a natural product was assessed through the study. Furthermore, the applicability of ILs as crystal engineering solvents are expected to modify both the solid state and the crystal morphology of natural compounds, which can influence drug manufacturability, dissolution rate, and bioavailability.
\end{abstract}

Keywords: ionic liquid; natural product; crystallization; sucrose; crystal morphology

\section{Introduction}

Crystallization is a solid-liquid separation technique for extracting solutes from the solution under their crystalline form. The technique is characterized by molecular recognition and self-assembly 
principles. Crystals obtained through crystallization have different crystal structures, morphology, particle distribution, purity, yield, and so forth. Therefore, crystallization techniques are widely applied by pharmaceutical or fine chemical industries in order to produce their final product in solid form [1,2]. Until now, studies regarding the application of crystallization techniques for the separation of single compounds from natural sources have been significant.

Crystal morphological characteristics could have an impact on the safety, efficacy, and quality of drug products [3]. It is well known that the control of crystal morphology, as well as the transformation to amorphous forms of a solid-state material, greatly influence the manufacturability, dissolution rate, and bioavailability of most active pharmaceutical ingredients (APIs) [4]. Moreover, downstream processes, including washing, drying, milling, and so on, are hindered by specific crystal morphology, such as a needle-like morphology. Furthermore, some particular crystal morphologies are known to cause poor flowability, inadequate mixing, compacting, and adhesion properties, which can consequently overburden the drug tableting and manufacturing processes [5]. To put in another way, the change in the crystal morphology and solid state can improve the drug bioavailability and can also ease the manufacturing process.

Ionic liquids (ILs) are salts characterized by a melting point below $100{ }^{\circ} \mathrm{C}$. They are composed of anions and cations which function individually and separately as ions while maintaining the characteristics of ILs. ILs are referred as the "design solvents" because the physiochemical characteristic can be altered through different combinations of cation and anion. General properties of ILs include high thermal stability, non-volatility, low vapor pressure, high dissolution capacity of organic and inorganic components, large liquid range, high polarity, high ion conductivity, etc. [6,7].

Based on the unique properties of ILs, Reichert et al. [8] reported the design of crystallization techniques with the application of ILs as crystallization solvents in their review. It was suggested that, compared with generally-applied crystallization techniques, crystallization can be induced in a wide range of variables when ILs are applied as crystallization solvents. However, studies involving ILs as crystallization solvents have encountered some limitations due to ILs' expensive unit price following the exceptional synthesis process and the existence of nearly $10^{18}$ ionic liquid combinations. Nevertheless, despite all these difficulties, presently, several studies involving the use of ILs in crystallization have been vigorously conducted [9-16]. Furthermore, studies involving ILs in crystallization have been generally carried out to evaluate the effect of ILs as precipitating agents or as additives in protein crystallization [17-20]. Currently, a study suggesting the effect of ILs as solvents suitable to control $\mathrm{CaCO}_{3}$ polymorphs has been reported [21]. Additionally, the potential application of ILs for the polymorph design and control of APIs was suggested by Stoimenovski et al. [22]. In a recent investigation, An et al. [23-26] assessed the effectiveness of ILs as promising solvents for the design and control of APIs' polymorphs. In addition, a study suggesting ILs as crystal engineering solvents for the design of new crystal structure of nicotinamide/oxalic acid salt (2:1) [27] as well as a study regarding the use of ILs in supramolecular synthesis [28] have been reported.

Research on natural product crystallization was reported in 2006 by Lapkin et al. [29]; here, with the use of ILs, they were able to achieve crystallization of artemisinin, an anti-malarial agent, from the medicinal plant, Artemisia annua, with $95 \%$ purity and $82 \%$ yield. Further, in their study, Ventura et al. [30] suggested ILs as efficient materials to extract and to isolate bioactive compounds. However, additional research on the use of ILs for natural product crystallization are still insignificant. Efforts to resolve the low quality and efficacy drawbacks of conventional methods of natural product isolation techniques are yet miniscule, thus, impacting the slow application of the potential alternative green solvent ILs.

Angelica gigas Nakai (A. gigas) is a Korean medicinal herb which contains coumarins, polysaccharides, as well as volatile aromatic compounds. In Korean folk medicine, A. gigas is utilized to treat hypertension, anemia, and gynecological disorders. It is also used as sedative, pain reliever, tonic, and so on. Coumarins, including decursin and decursinol angelate, are the main bioactive compounds in this herb. Generally, A. gigas is extracted with methanol and then followed by several 
chromatographic techniques to isolate single active components from the plant [31-33]. However, these techniques are tedious, time-consuming, and produce products of poor quality. Thus, the application of a new solvent and efficient separation techniques are assumed as an absolute necessity to enhance the extraction and separation methods for the production of single compounds with high purity from A. gigas. Accordingly, this study was carried out to investigate the applicability of crystallization techniques for the isolation of natural product, as well as to evaluate the efficacy of ILs in natural product crystallization.

In this study, methanol $(\mathrm{MeOH})$ was used for the extraction of the medicinal herb namely A. gigas. Anti-solvent, including ILs $\left(\mathrm{BMImBF}_{4}, \mathrm{BMImPF}_{6}, \mathrm{BMImTFSI}^{\mathrm{AEImBF}} 4\right.$, and $\left.\mathrm{AAImBF}_{4}\right)$ and organic solvents (n-hexane, methylene chloride, acetone, and acetonitrile) were individually added to the methanol extraction solution at respectively 1:1, 1:2, and 1:3 (extraction solution/anti-solvent $(v / v)$ ) ratio to induce crystallization. The mixtures were allowed to settle for three days to evaluate the crystal formation. As a result, even after three days, crystals were not obtained when organic solvents were used as an anti-solvent. However, crystals were educed within less than $8 \mathrm{~h}$ when using ILs (Figure 1).

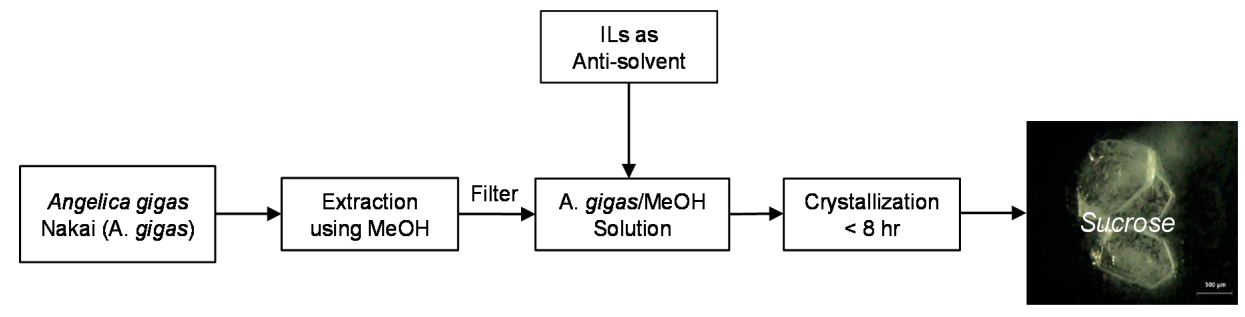

Figure 1. The selective crystallization separation procedure diagram for the isolation of sucrose form the methanol extract of $A$. gigas using Ionic liquids.

The collected crystals were analyzed and confirmed as crystals of sucrose by means of ${ }^{1} \mathrm{H}-\mathrm{NMR}$ (A. gigas extraction solution/ILs: $3 \mathrm{~mL} / 3 \mathrm{~mL}(1: 1 \mathrm{v} / \mathrm{v})$, Figure 2). The ${ }^{1} \mathrm{H}-\mathrm{NMR}$ result of the collected sucrose crystals was compared to the one analyzed and published by Ferreira et al [34].

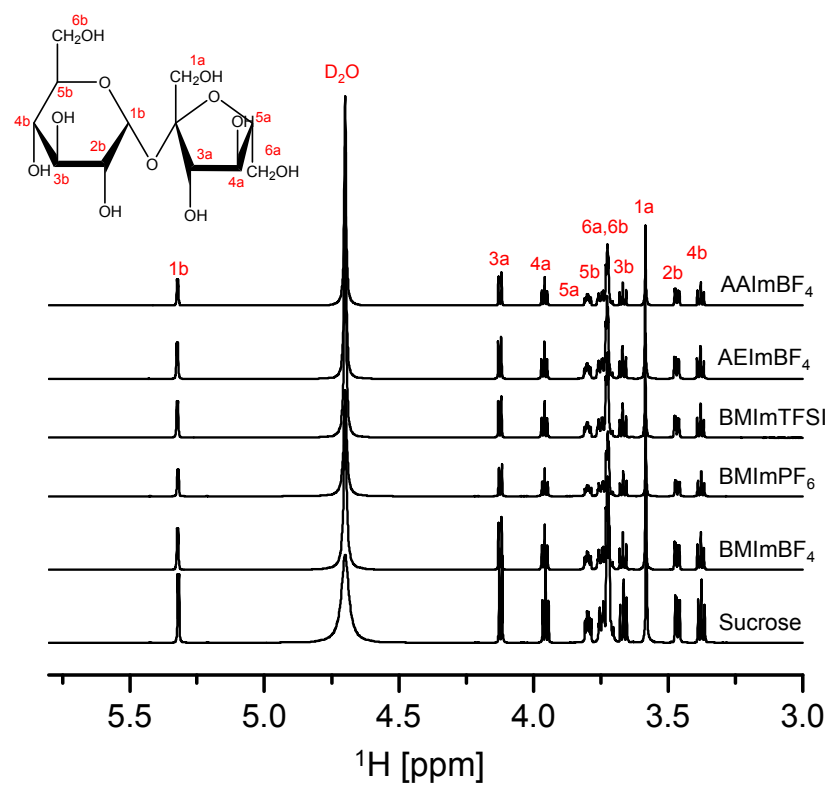

Figure 2. ${ }^{1} \mathrm{H}-\mathrm{NMR}$ spectrum of sucrose crystals precipitated from the $\mathrm{MeOH}$ extraction solution of A. gigas when utilizing ILs as anti-solvent (A. gigas extraction solution/ILs: $3 \mathrm{~mL} / 3 \mathrm{~mL}(1: 1 \mathrm{v} / v)$, solvent: $\left.\mathrm{D}_{2} \mathrm{O}\right)$. 
Further, the crystal structure of the obtained sucrose crystals was analyzed and determined by means of single-crystal X-ray diffraction (SXD) as well as by PXRD patterns. The change in crystal morphology was monitored via the video microscope.

Accordingly, this work aims to report the crystal structure and the changes in morphology of sucrose crystals collected from the $\mathrm{MeOH}$ extract of $A$. gigas when an ionic liquid was used as the anti-solvent.

\section{Results and Discussion}

The Table 1 illustrates the SXD unit cell data of sucrose crystals precipitated from the A. gigas extraction solution/ILs: $3 \mathrm{~mL} / 3 \mathrm{~mL}(1: 1 v / v)$. The results revealed that all the sucrose crystals obtained when $\mathrm{BMImBF}_{4}, \mathrm{BMImPF}_{6}, \mathrm{BMImTFSI} \mathrm{AEImBF}$, and $\mathrm{AAImBF}_{4}$ ILs were employed belong to the monoclinic system and $P 2_{1}$ space group. Moreover, the crystal axes and angles were identical for all crystals. The data presented coincide with the unit cell data of sucrose crystals suggested in 1972 by Brown and Levy [35].

Table 1. Single-crystal X-ray diffraction (SXD) data of sucrose crystals isolated from A. gigas.

\begin{tabular}{cccccc}
\hline Ionic Liquids (3mL) & $\mathbf{B M I m B F}_{\mathbf{4}}$ & $\mathbf{B M I m P F}_{\mathbf{6}}$ & $\mathbf{A A I m B F}_{\mathbf{4}}$ & $\mathbf{A E I m B F}_{\mathbf{4}}$ & BMImTFSI \\
\hline $\mathrm{a}(\AA)$ & 7.751 & 7.734 & 7.778 & 7.760 & 7.767 \\
$\mathrm{~b}(\AA)$ & 8.775 & 8.697 & 8.722 & 8.712 & 8.725 \\
$\mathrm{c}(\AA)$ & 10.804 & 10.826 & 10.884 & 10.853 & 10.883 \\
$\beta\left({ }^{\circ}\right)$ & 103.060 & 103.006 & 102.967 & 102.946 & 102.912 \\
Cell Vol. $\left(\AA^{3}\right)$ & 715.8 & 709.5 & 719.5 & 715.1 & 718.9 \\
Space group & $P 2_{1}$ & $P 2_{1}$ & $P 2_{1}$ & $P 2_{1}$ & $P 2_{1}$ \\
$Z$ & 2 & 2 & 2 & 2 & 2 \\
Crystal system & monoclinic & monoclinic & monoclinic & monoclinic & monoclinic \\
\hline
\end{tabular}

Therefore, we could forecast that sucrose crystals isolated from A. gigas when ILs were used as the anti-solvent have identic crystal structure as the previously-reported sucrose crystals. For more specific analysis with regard to SXD analysis results, the crystal packing structure of sucrose crystals educed by BMImBF 4 was studied. The molecule conformation structure of sucrose crystals made with $\mathrm{BMImBF}_{4}$ is depicted in Figure 3. According to the molecular structure data, the conformation and the packing of sucrose molecules were perceived and the crystal structure could be achieved.

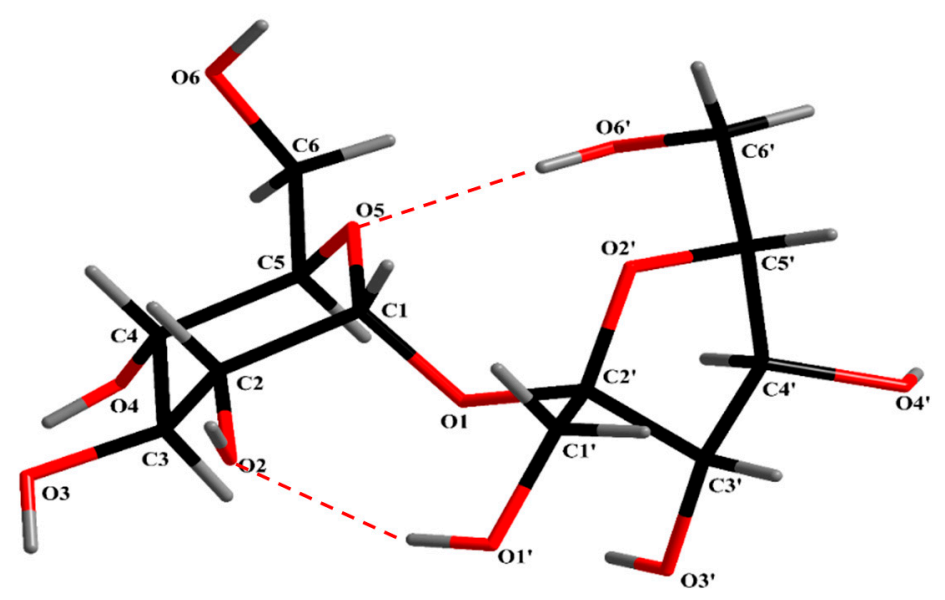

Figure 3. Conformational structure of sucrose molecule isolated from the methanol extract of A. gigas when $\mathrm{BMImBF}_{4}$ was used as the anti-solvent. 
Table 2 demonstrates the intermolecular and intramolecular hydrogen bonding between functional groups of sucrose crystals educed when $\mathrm{BMImBF}_{4}$ was employed. The intramolecular hydrogen bonds presented in Table 2 are observed between $\mathrm{O}^{\prime}(1)-\mathrm{H} \cdots \mathrm{O}(2)$ and $\mathrm{O}^{\prime}(6)-\mathrm{H} \cdots \mathrm{O}(5)$, as illustrated in Figure 3. Moreover, the intermolecular hydrogen bonding presented is shown in the crystallographic structure in Figure 4a. Additionally, the arrangement of sucrose crystals educed from BMImBF 4 is represented in Figure $4 \mathrm{~b}$. These sucrose crystal structures are in good accordance with the sucrose crystal structure proposed by Brown and Levy [35]. Thus, we could confirm that all sucrose crystals precipitated using the process depicted in Figure 1 have similar crystal structure and coincide with the sucrose crystal structure data suggested by Brown and Levy in 1972. The sucrose intermolecular hydrogen bonds $(\alpha, \beta, \gamma, \delta, \varepsilon)$ illustrated in Table 2 were identified to be periodic bonds chains (PBCs) by Brown and Levy [35], as well as by Aquilano et al [36]. According to the Hartman-Perdok theory of the PBCs concept, crystal faces involved in crystal growth are classified as follows: F (flat) faces are those having more than two PBCs; faces with only one PBC are called S (stepped) faces; and those without PBC are called $\mathrm{K}$ (kinked) faces. Here, it was suggested that the crystal growth rate is fast at $\mathrm{K}$ faces, intermediate at $\mathrm{S}$ faces, and slow at $\mathrm{F}$ faces. Additionally, it was suggested that the control of the crystal morphology without the alternation of its crystal structure is possible [37] and the F faces are involved in the process.

Table 2. Hydrogen bonds of sucrose separated from the methanol extract of $A$. gigas using ionic liquids.

\begin{tabular}{cc}
\hline Intramolecular & Intermolecular Periodic Bonds Chains (PBCs) \\
\hline & $\alpha: \mathrm{O}(3)-\mathrm{H} \cdots \mathrm{O}^{\prime}(3)$ \\
$\mathrm{O}^{\prime}(1)-\mathrm{H} \cdots \mathrm{O}(2)$ & $\beta: \mathrm{O}(6)-\mathrm{H} \cdots \mathrm{O}(3)$ \\
$\mathrm{O}^{\prime}(6)-\mathrm{H} \cdots \mathrm{O}(5)$ & $\gamma: \mathrm{O}(2)-\mathrm{H} \cdots \mathrm{O}^{\prime}(6)$ \\
& $\delta: \mathrm{O}^{\prime}(3)-\mathrm{H}^{\prime} \cdots \mathrm{O}^{\prime}(4)$ \\
& $\varepsilon: \mathrm{O}^{\prime}(4)-\mathrm{H}^{\prime} \cdots \mathrm{O}^{\prime}(1)$ \\
\hline
\end{tabular}
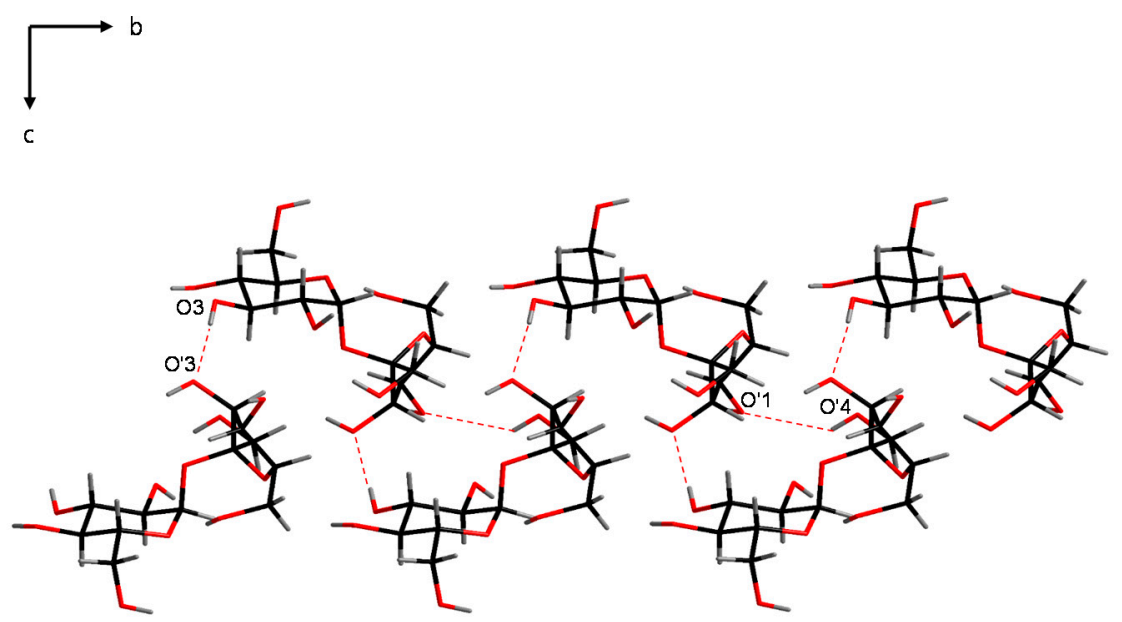

(a)

Figure 4. Cont. 


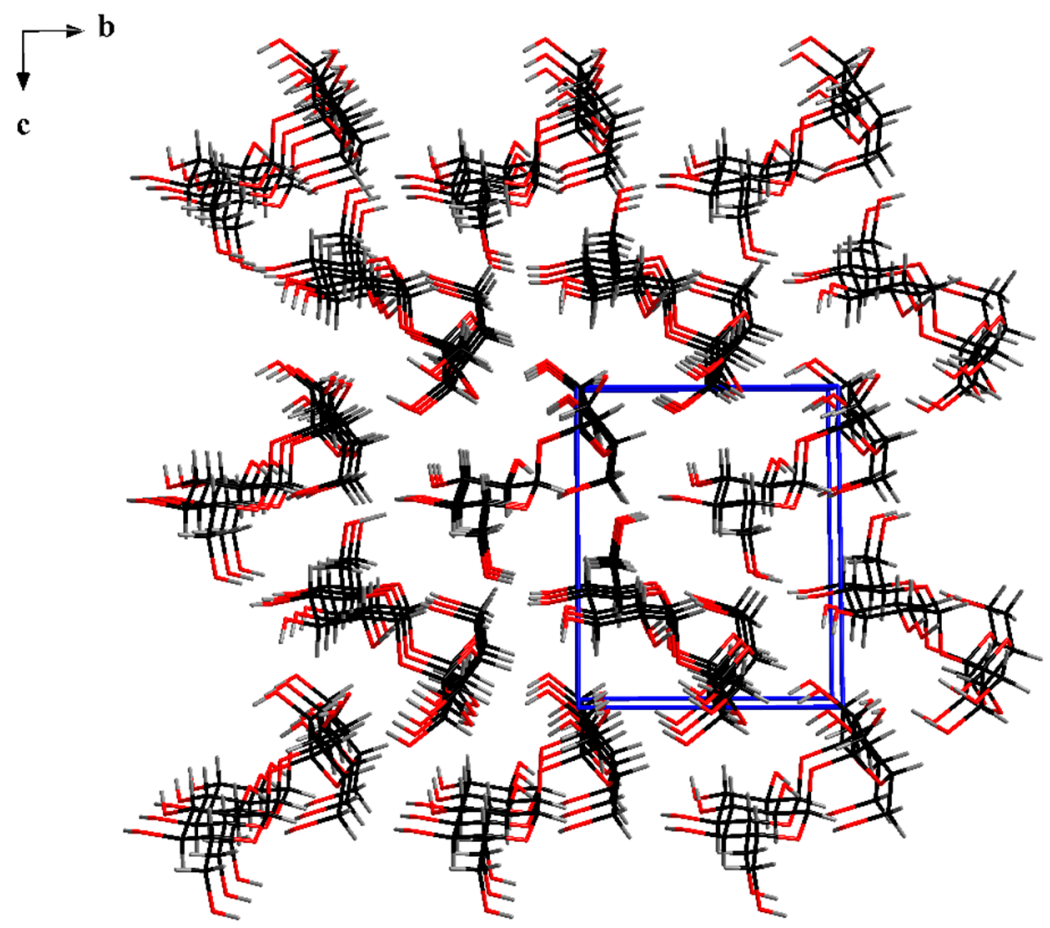

(b)

Figure 4. The crystal structure of the sucrose separated from the methanol extract of $A$. gigas when $\mathrm{BMImBF}_{4}$ IL was used as an anti-solvent: (a) Crystal packing of six sucrose molecules, (b) Sucrose crystal packing structure.

The morphology of sucrose crystals educed from the $\mathrm{MeOH}$ extract of $A$. gigas at different A. gigas extraction solution/ILs $(v / v)$ ratios was monitored by means of video microscope $(300 \times)$. According to the result, the change in crystal morphology was observed with the variation of the type and the concentration of ILs. Additionally, these sucrose crystals were found to possess distinct morphologies compared with the currently-marketed sucrose crystals, which have large rectangular shapes (Figure S1). In Figure 5 the images of the sucrose crystal morphologies obtained according the $\mathrm{BMImBF}_{4}$ concentration variation are illustrated. When $3 \mathrm{~mL}$ of $\mathrm{BMImBF}_{4}(A$. gigas extract solution/ $\mathrm{BMImBF}_{4}(1: 1(v / v))$ was used, the sucrose crystals were long and hexagon-shaped. However, at $6 \mathrm{~mL}(A$. gigas extract solution/BMImBF $4(1: 2(v / v))$ and $9 \mathrm{~mL}(A$. gigas extraction solution/BMImBF $4(1: 3(v / v)) \mathrm{BMImBF}_{4}$ composition, long, rectangular-shaped sucrose crystals were produced. Further, it was confirmed that all sucrose crystals possessed identic PXRD patterns regardless of the concentration of $\mathrm{BMImBF}_{4}$ (Figure S2).
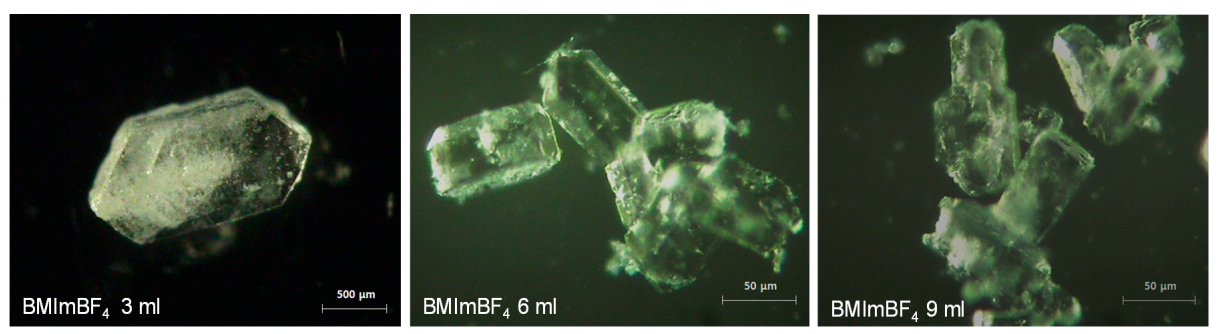

Figure 5. Video-microscope images of sucrose crystals obtained from the $\mathrm{MeOH}$ extraction solution of $A$. gigas (solvent volume of $3 \mathrm{~mL}$ ) under $\mathrm{BMImBF}_{4}$ anti-solvent concentration change using crystallization techniques. 
In addition, $\mathrm{AEImBF}_{4}$ and $\mathrm{AAImBF}_{4}$, ILs characterized by an identical anion, $\mathrm{BF}_{4}{ }^{-}$, but with cations of distinct alkyl chains, were compared with $\mathrm{BMImBF}_{4}$. Here, sucrose crystals obtained from $\mathrm{AEImBF}_{4}$ and $\mathrm{AAImBF}_{4}$ possessed the identical morphology with those precipitated with $\mathrm{BMImBF}_{4}$ (Figure S4). Figure 6 depicts various crystal shapes of sucrose crystals precipitated from $\mathrm{BMImPF}_{6}$ under IL concentration change. When $\mathrm{BMImPF}_{6}$ composition was $3 \mathrm{~mL}$ (A. gigas extraction solution/BMImPF $6(1: 1(v / v))$, elongated hexagon-shaped sucrose crystals were produced. Subsequently, long, rectangular-shaped crystals and small, rectangular-shaped sucrose crystals were observed at $6 \mathrm{~mL}$ (A. gigas extract solution/BMImPF $6(1: 2(v / v))$ and $9 \mathrm{~mL}$ (A. gigas extraction solution $/ \mathrm{BMImPF}_{6}(1: 3(v / v)) \mathrm{BMImPF}_{6}$ composition, respectively. Regardless of their shapes, all sucrose crystals precipitated from $\mathrm{BMImPF}_{6}$ were found to possess identical RXRD patterns (Figure $\mathrm{S} 3$ ).
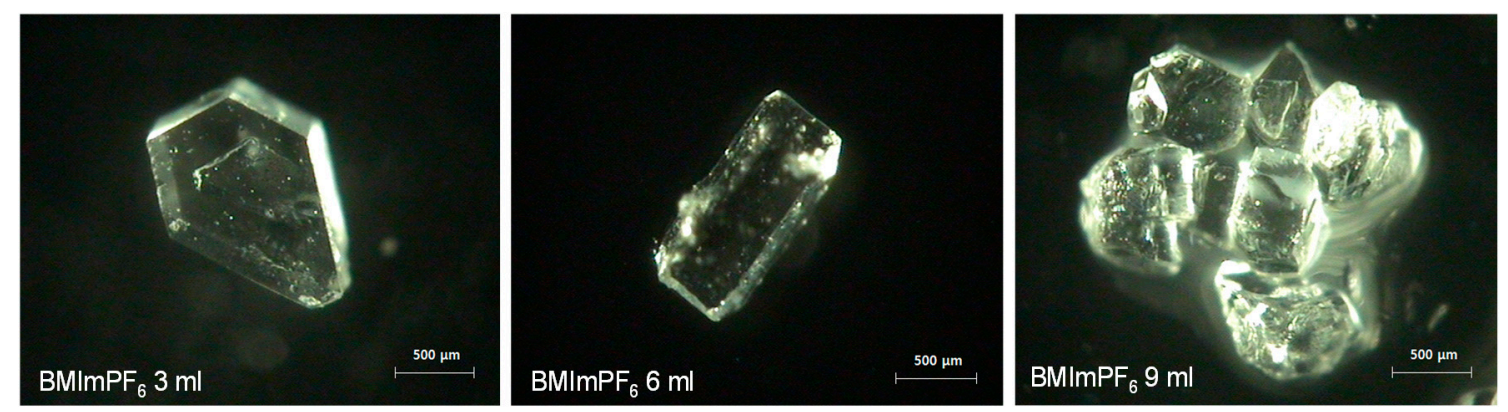

Figure 6. Video microscope images of sucrose crystals precipitated from the $\mathrm{MeOH}$ extraction solution of A. gigas (solvent volume of $3 \mathrm{~mL}$ ) at varied concentrations of $\mathrm{BMImPF}_{6}$ anti-solvent changes using crystallization techniques.

Sucrose crystals precipitated using BMImTFSI presented different crystal morphologies and PXRD patterns compared to the others. Figure 7 illustrates various morphologies of sucrose crystals precipitated under BMImTFSI concentration change. As a result, under BMImTFSI $3 \mathrm{~mL}$ and $6 \mathrm{~mL}$ (A. gigas extraction solution/BMImTFSI $(1: 1(v / v))$ and $(1: 2(v / v))$ conditions, sucrose crystals were characterized by a large, rectangular-shaped crystals with small, rectangular-shaped crystals formed on the surface due to the secondary nucleation and growth phenomenon [38]. However, crystals obtained with BMImTFSI $9 \mathrm{~mL}$ (A. gigas extraction solution/BMImTFSI $(1: 3(v / v))$ were confirmed to be amorphous sucrose crystals. Moreover, identical patterns of PXRD were observed for the sucrose crystals obtained at $3 \mathrm{~mL}$ and $6 \mathrm{~mL}$ of BMImTFSI and those at BMImBF 4 and BMImPF 6. However, $^{2}$ sucrose crystals precipitated from $9 \mathrm{~mL}$ of BMImTFSI were confirmed to be amorphous according to the PXRD patterns (Figure 8). Also, DSC curves with onset temperature at approximately $182{ }^{\circ} \mathrm{C}$ and endothermic temperature at $191^{\circ} \mathrm{C}$ was recorded for sucrose crystals obtained from BMImTFSI $3 \mathrm{~mL}$ and $6 \mathrm{~mL}$ (DSC experiment conditions: heating rate: $10^{\circ} \mathrm{C} / \mathrm{min}$ and the temperature range set from $30^{\circ} \mathrm{C}$ to $230^{\circ} \mathrm{C}$ ). Nevertheless, neither endothermic nor exothermic peaks could be observed from the DSC curve of sucrose crystals precipitated from BMImTFSI $9 \mathrm{~mL}$ (Figure S5). Therefore, these sucrose crystals were confirmed as amorphous materials. The precipitation of amorphous material is assumed to be a result of the provocation of a rapid increase in supersaturation level by addition of a large amount of BMImTFSI which led to the consequent production of amorphous sucrose. Further, the simulated PXRD patterns and analytical PXRD patterns of sucrose crystals precipitated from ILs were found to be identical (Figure 8). 

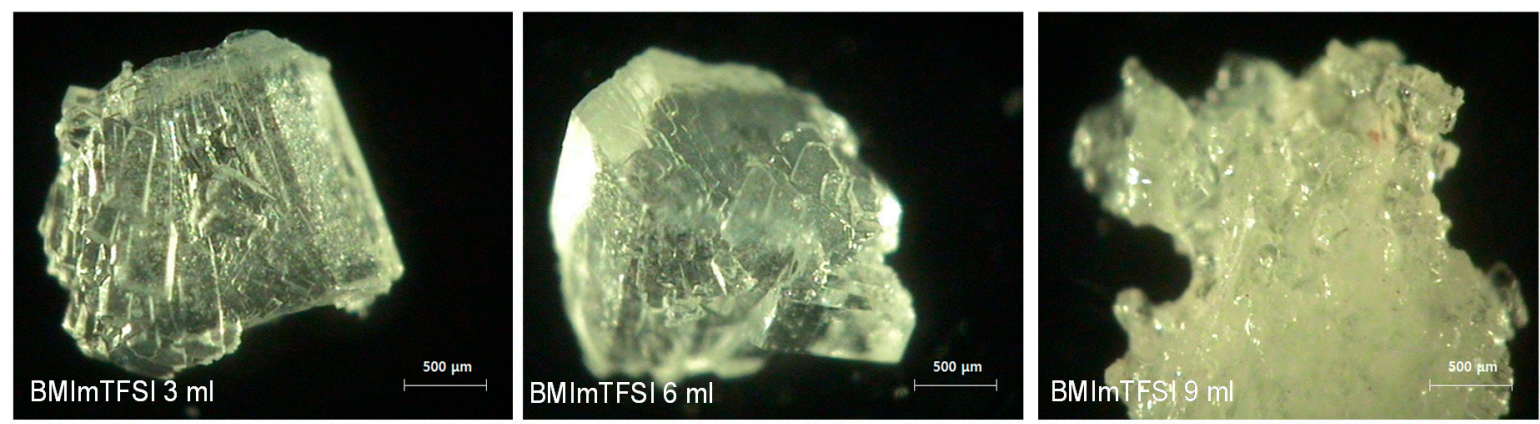

Figure 7. Video microscope images of sucrose crystals produced from the $\mathrm{MeOH}$ extraction solution of A. gigas (solvent volume of $3 \mathrm{~mL}$ ) under BMImTFSI anti-solvent concentration change conditions using crystallization methods.

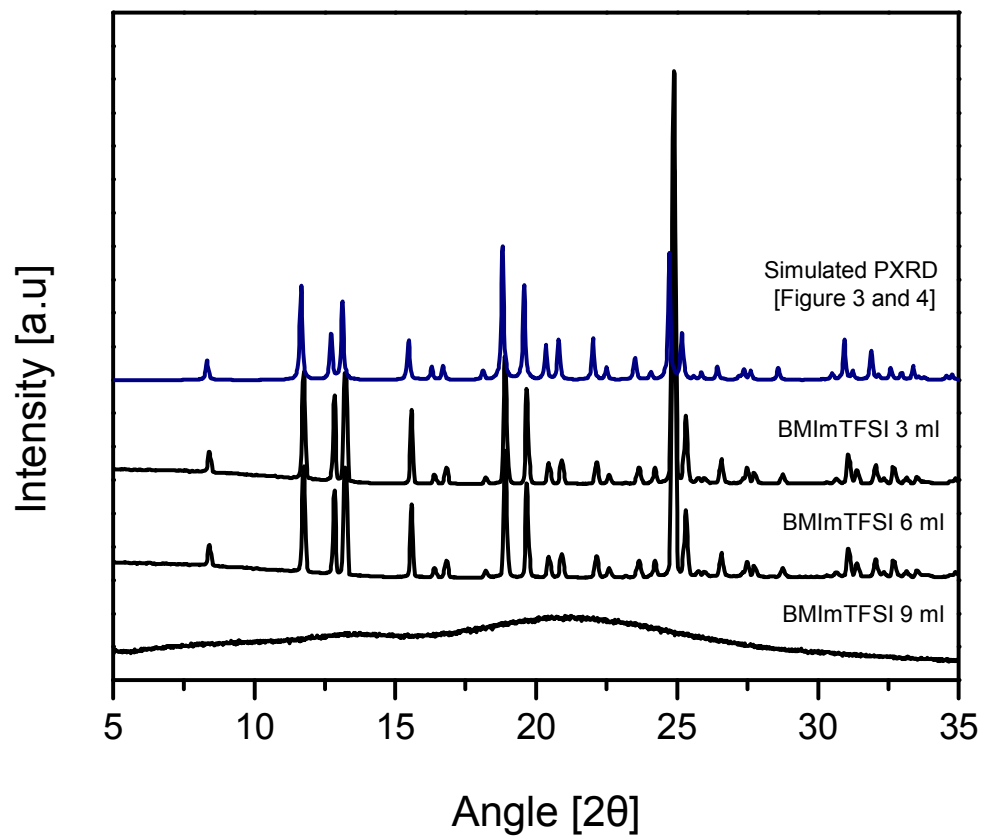

Figure 8. PXRD patterns of sucrose crystals precipitated from the $\mathrm{MeOH}$ extraction solution of A. gigas at a constant solvent volume of $3 \mathrm{~mL}$ under BMImTFSI anti-solvent composition change conditions.

As observed in Figures 3 and 4, crystals have similar structures, but different crystal morphologies; this is due to differences in the adhesion of additional solvent, impurities, additives, etc., to the crystal surface, or due to the differences in the supersaturation level [37]. This phenomenon occurs when crystals possess $\mathrm{F}$ faces with more than two PBCs. According to the report by Brown and Levy, the sucrose crystals have five different PBCs [35,36]. Moreover, it was confirmed that all sucrose crystals obtained in the present study have the same crystal structure with the sucrose crystals reported by Brown and Levy [35]. Therefore, it is conceivable that these sucrose crystals have a distinct crystal morphology.

In 2008, Li et al. carried out a study on the crystallization of the lysozyme protein using $\mathrm{BMImBF}_{4}$ as an additive; here, it was found that lysozyme had a different crystal morphology depending on the addition of ILs. They assumed the $\mathrm{BF}_{4}{ }^{-}$, the anion of ILs, adhered to the surface of the lysozyme crystal, which resulted in the obstruction of the crystal growth [20]. We presumed that the variation of crystal morphology of sucrose crystals precipitated under different ionic liquid conditions has similar aspects with the result reported by Li et al. It could be concluded as such because the anion was the variable, and the cation was the control in this study. The ILs used in the experiment had a imidazolium 
cation base with the only variation in functional groups. However, three different types of anions, $\mathrm{BF}_{4}^{-}, \mathrm{PF}_{6}{ }^{-}, \mathrm{TFSI}^{-}$, were included. Nevertheless, different crystal morphologies could be observed for crystals precipitated from ILs composed with distinct anions, such as $\mathrm{BF}_{4}{ }^{-}, \mathrm{PF}_{6}{ }^{-}, \mathrm{TFSI}^{-}$, as can be seen in Figures 5-7. ${ }^{19} \mathrm{~F}-\mathrm{NMR}$ analysis was conducted on sucrose crystals depicted in Figures 5 and 6 (when $\mathrm{BMImBF}_{4} 3 \mathrm{~mL}$ and $\mathrm{BMImPF}_{6} 3 \mathrm{~mL}$ were used as the anti-solvent) to demonstrate in detail whether the ILs anions including $\mathrm{BF}_{4}{ }^{-}, \mathrm{PF}_{6}{ }^{-}$, and so on, are attached on the crystals' surfaces. The ${ }^{19} \mathrm{~F}-\mathrm{NMR}$ results are illustrated in Figure 9. As a result, a ${ }^{19} \mathrm{~F}-\mathrm{NMR}$ spectrum consisting of two singlets corresponding to $\mathrm{F}^{-}$observed at $-150 \mathrm{ppm}$ in approximately 1:4 ratio was recorded for sucrose crystals precipitated from BMImBF 3 mL (Figure 9a). The spectrum coincided with the BMImBF 4 ${ }^{19} \mathrm{~F}-\mathrm{NMR}$ spectrum (Figure $9 \mathrm{~b}$ ). Moreover, the ${ }^{19} \mathrm{~F}-\mathrm{NMR}$ spectrum corresponding to sucrose crystals precipitated from BMImBF 3 mL, illustrated in Figure 9a was affirmed to be similar with ${ }^{19} \mathrm{~F}-\mathrm{NMR}$ spectrum of $\mathrm{BF}_{4}{ }^{-}$anion reported by Wiemers-Meyer et al [39]. Based on the result depicted in Figure 9, we could presume that not $\mathrm{BMIm}^{+}$, but only $\mathrm{BF}_{4}{ }^{-}$, were attached on the surfaces of sucrose crystals. Additionally, it is assumed that, the peak of the imidazolium base would be observed on the ${ }^{1} \mathrm{H}-\mathrm{NMR}$ spectra (Figure 2) if the crystals were not washed and some ILs remained on the surface. However, on the present ${ }^{1} \mathrm{H}-\mathrm{NMR}$ spectra in Figure 2 only the peaks of pure sucrose could be identified. Accordingly, we presume that $\mathrm{BMIm}^{+}$was well removed from the crystals during the washing process and only the $\mathrm{BF}_{4}{ }^{-}$anions are still present on the crystals' surfaces. Additionally, it has been reported that $\mathrm{BF}_{4}{ }^{-}$ and $\mathrm{PF}_{6}{ }^{-}$hydrolyze to produce HF [40]. Based on the above report, the result in Figure 9 can be misinterpreted as not $\mathrm{BF}_{4}^{-}$, but $\mathrm{F}^{-}$, bound on the surface of sucrose crystals. Nonetheless, according to the literature, the peak of HF appears as a singlet which is randomly observed at $-17 \mathrm{ppm}$ or $-153 \mathrm{ppm}[40,41]$. As the ${ }^{19} \mathrm{~F}-\mathrm{NMR}$ spectrum of $\mathrm{F}^{-}$was dissimilar with the ${ }^{19} \mathrm{~F}-\mathrm{NMR}$ spectra illustrate in Figure 9, it is assumed that no $\mathrm{F}^{-}$remained bound on the surfaces of sucrose crystals. In addition, the ${ }^{19} \mathrm{~F}-\mathrm{NMR}$ spectrum of $\mathrm{BMImPF}_{6}$ consisted of two doublets corresponding to the $\mathrm{F}^{-}$observed at approximately $-70 \mathrm{ppm}$ and $-73 \mathrm{pm}$ (Figure S6). Moreover, the spectrum was found to coincide with $\mathrm{PF}_{6}{ }^{-}$spectrum reported by Plakhothyk et al. [40]. Nevertheless, no peak was identified on the ${ }^{19} \mathrm{~F}-\mathrm{NMR}$ spectrum of sucrose crystals produced from $\mathrm{BMImPF}_{6} 3 \mathrm{~mL}$ (Figure S7). Either the feeble binding energy of $\mathrm{PF}_{6}{ }^{-}$on the surface of sucrose crystals compared to $\mathrm{BF}_{4}{ }^{-}$, or the high miscibility of $\mathrm{BMImPF}_{6}$ in the washing solvent, $\mathrm{MeOH}$, is assumed as the reason leading to a complete removal of $\mathrm{PF}_{6}{ }^{-}$from the crystals during their washing process. Therefore, we suggest that the ILs' anions selectively adhere to distinct and particular surfaces ( $F$ faces) of sucrose crystals and hinder the crystal growth on the adhered surface, which results in differences in crystal morphology.

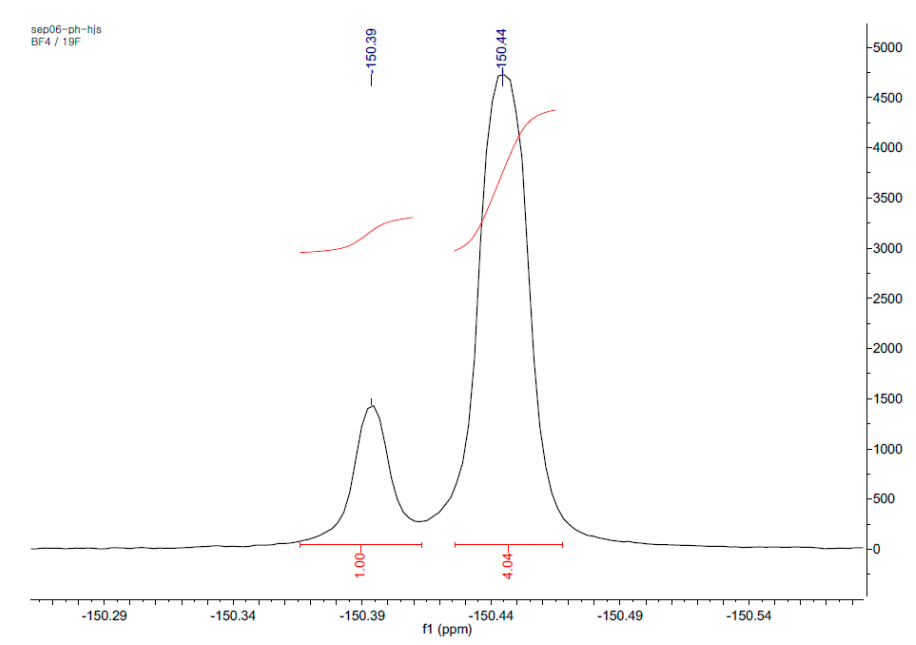

(a)

Figure 9. Cont. 


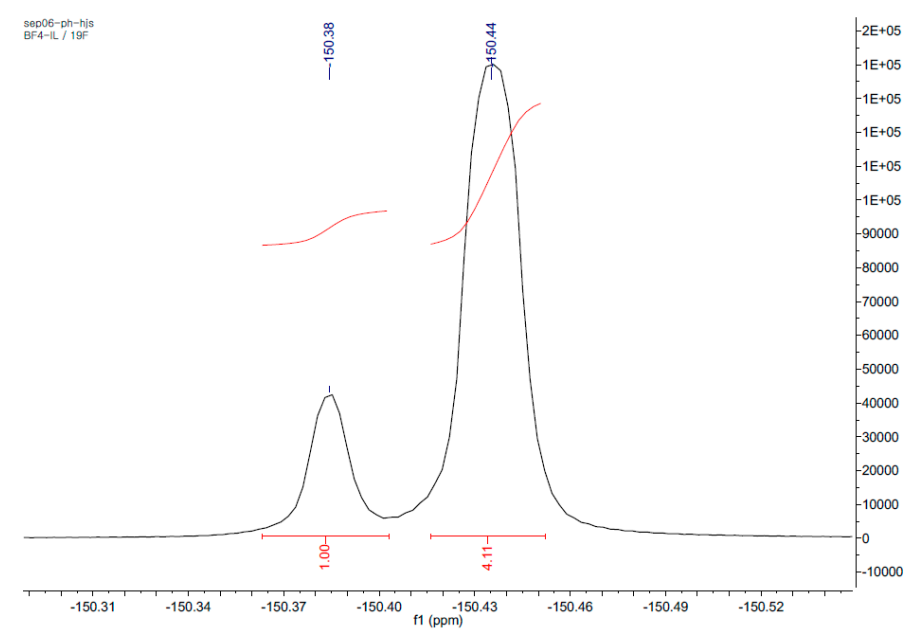

(b)

Figure 9. ${ }^{19} \mathrm{~F}-\mathrm{NMR}$ spectrum of sucrose crystals precipitated from $\mathrm{BMImBF}_{4}$. (A. gigas extraction solution/ILs: $3 \mathrm{~mL} / 3 \mathrm{~mL}(1: 1 v / v)$, solvent: $\left.\mathrm{D}_{2} \mathrm{O}\right)(\mathbf{a})$ sucrose crystal, and (b) $\mathrm{BMImBF}_{4}$.

With regards to the above results, ILs are expected as potential crystal engineering solvents for their ability to induce crystallization of natural compounds, as well as changing the crystal morphology and solid state. Additionally, ILs possess the potential of influencing and improving the bioavailability and manufacturability of produced products through natural product crystallization. Therefore, it is promising to utilize ILs as crystallization solvents for the selective separation of natural compounds from natural sources.

Subsequent to the fundamental experimental results, our ongoing research study aims to apply ILs as natural product crystallization solvents to isolate bioactive compounds from A. gigas. The follow-up research also intends to investigate the influence of ILs on the characteristic changes of the crystals of isolated bioactive compounds.

\section{Materials and Methods}

\subsection{Materials}

The Korean medicinal herb, A. gigas was collected from a local market in Seoul, Korea and identified by professor Young-Won Chin of Dongguk University. Ionic liquids, including 1-butyl-3methylimidazolium tetrafluoroborate $\left(\mathrm{BMImBF}_{4}\right)$, 1-butyl-3-methylimidazolium hexafluoro-phosphate $\left(\mathrm{BMImPF}_{6}\right.$ ), and 1-butyl-3-methylimidazolium bis(trifluoromethylsulfonyl)imide (BMImTFSI) were purchased from A-star Co., Ltd. (Ulsan, Korea), and, 1-allyl-3-ethylimidazolium tetrafluoroborate $\left(\mathrm{AEImBF}_{4}\right)$ and 1,3-diallylimidazolium tetrafluoroborate $\left(\mathrm{AAImBF}_{4}\right)$ were purchased from Kanto Chem Co., Ltd. (Tokyo, Japan).

\subsection{Crystallization Process of Sucrose}

Pulverized dried roots of $A$. gigas ( $300 \mathrm{mg}$ ) and $5 \mathrm{~mL}$ of $\mathrm{MeOH}$ were placed in a $20 \mathrm{~mL}$ vial. Thereafter, the system was agitated at $200 \mathrm{rpm}$ and $25^{\circ} \mathrm{C}$ for $1 \mathrm{~h}$. Afterwards, the methanol extract was vacuum filtered and the filtrate was collected. Several samples of the $\mathrm{MeOH}$ extract of A. gigas $(3 \mathrm{~mL}$ each) were prepared for the following process. Subsequently, anti-solvents, including ILs BMImBF 4 , $\mathrm{BMImPF}_{6}, \mathrm{BMImTFSI}^{\mathrm{AEImBF}}$, and $\mathrm{AAImBF}_{4}$, and organic solvents n-hexane, methylene chloride, acetone, and acetonitrile, were individually added to samples at different volumes of 3,6 , and $9 \mathrm{~mL}$ (extract solution/anti-solvent ratios 1:1, 1:2, 1:3 (v/v)). Each mixture was stirred at $25^{\circ} \mathrm{C}$ and $100 \mathrm{rpm}$ for three days, maximum, then filtered to collect the crystals. As a result, when the organic solvents were used as anti-solvents, no crystals were obtained even after three days of the experiment. However, 
crystals were educed within less than $8 \mathrm{~h}$ with ILs as anti-solvents. Afterwards, produced crystals were subjected to vacuum filtration and were washed with $5 \mathrm{~mL}$ of $\mathrm{MeOH}$ three times while being filtered. The collected crystals were analyzed by means of ${ }^{1} \mathrm{H}-\mathrm{NMR}, \mathrm{SXD}$, and video-microscope and were confirmed to be single crystals of sucrose molecules. Accordingly, the experiment process chart has been suggested in Figure 1.

\subsection{Nuclear Magnetic Resonance Spectroscopy (Solution-State NMR)}

The ${ }^{1} \mathrm{H}-\mathrm{NMR}$ and ${ }^{19} \mathrm{~F}-\mathrm{NMR}$ ((solvent: $\left.\mathrm{D}_{2} \mathrm{O}\right) 800 \mathrm{MHz}$ and $300 \mathrm{MHz}$ FT-NMR, Bruker, MA, USA) analysis were carried out to confirm the molecular structure of sucrose crystals isolated from the methanol extract of $A$. gigas.

\subsection{Powder X-ray Diffraction (PXRD)}

PXRD analysis was conducted on a powder X-ray diffractometer (D8 Advance, Bruker, Billerica, MA, USA) equipped with a $\mathrm{Cu} \mathrm{K} \alpha$ radiation source. The analysis was operated at $45 \mathrm{kV}$ and $40 \mathrm{~mA}$. The divergence slit and anti-scatter slit were fixed at $1^{\circ}$, while the receiving slit was set at $0.2 \mathrm{~mm}$. The continuous scanning of sucrose crystals was performed at a range of $2 \theta$ values set from $5^{\circ}$ to $35^{\circ}$ with a scan rate of $3^{\circ} / \mathrm{min}\left(0.4 \mathrm{~s} / 0.02^{\circ}\right.$ interval).

\subsection{Morphological Study}

In order to investigate the morphology of sucrose crystals, images were taken by a video-microscope (SV-55, Sometech, Sungnam, Korea) at 300× magnifications.

\subsection{Single-Crystal X-ray Diffraction (SXD)}

A Rigaku R-AXIS RAPID diffractometer (Rigaku Corporation, Tokyo, Japan) equipped with Monochromatic Mo-K $\alpha$ radiation $(\lambda=0.71073 \AA)$ and $3 \mathrm{~kW}$ was used for the single-crystal X-ray diffraction (SXD) analysis of single sucrose crystals. Diamond 3.2k software (Crystal Impact GbR, Bonn, Germany) was used for crystal packing plots.

\section{Conclusions}

The current study was conducted to investigate the efficacy of ionic liquids on the crystallization of a natural product. Here, the medicinal herb, A. gigas was extracted with $\mathrm{MeOH}$. Afterward, crystallization was induced with the use of various anti-solvents, including ILs and organic solvents, in order to compare their effects. The results showed that crystallization was not induced when organic solvents were used as anti-solvents. However, it was observed that when ILs were used, sucrose crystals could be obtained. Moreover, the SXD analysis result of sucrose crystals, isolated from the $\mathrm{MeOH}$ extract of $A$. gigas under different ionic liquid conditions, demonstrating that all sucrose crystals have identic crystal structure and revealed their structural similarity with currently-reported sucrose crystals. However, depending on the ionic liquid types, sucrose crystals displayed a distinct morphology. With regard to the theory of crystal habit, the difference in sucrose crystal morphology is assumed to be a result of distinctive adherence of ILs on the crystallographic surfaces, F faces, which disrupts the growth of the crystal, and consequently leads to the change of the morphology. Additionally, sucrose crystals obtained at a 1:3 (A. gigas extraction solution/BMImTFSI $(v / v)$ ) ratio were found to be amorphous. It is assumed that addition of a large amount of BMImTFSI anti-solvent provoked a great increment in sucrose supersaturation level, which consequently leads to the precipitation of amorphous sucrose crystals. It can be concluded that ILs played both a role of an anti-solvent to induce crystallization of natural products as well as a role of an additive for the control of the crystal morphology.

The change in crystal characteristics can positively impact the manufacturability, dissolution rate, and bioavailability of natural compounds, as well as the downstream processes, including filtration, 
washing, drying, milling, and other processes conducted after the crystallization step. Therefore, the potential of ILs as crystal engineering solvents could be expected.

Supplementary Materials: The following are available online at www.mdpi.com/2073-4352/7/10/284/s1, Figure S1. Crystal morphology of the currently commercialized sucrose crystals recorded by using the video microscope; Figure S2. PXRD patterns of sucrose crystals isolated from the MeOH extraction solution of $A$. gigas under controlled solvent volume $(3 \mathrm{~mL})$ and $\mathrm{BMImBF}_{4}$ anti-solvent concentration change conditions, using crystallization techniques; Figure S3. PXRD patterns of sucrose crystals produced from the MeOH extraction solution of $A$. gigas at constant solvent volume of $3 \mathrm{~mL}$ under $\mathrm{BMImPF}_{6}$ anti-solvent composition variation conditions using the crystallization method; Figure S4. Video-microscope photographs of sucrose crystals obtained from the $\mathrm{MeOH}$ extraction solution of $A$. gigas (solvent volume of $3 \mathrm{~mL}$ ) using, respectively, $\mathrm{BMImBF}_{4}$, $\mathrm{AEImBF}_{4}$, and $\mathrm{AAImBF}_{4}$ as anti-solvents $(A$. gigas extraction solution /ILs $(1: 1 \mathrm{v} / \mathrm{v})$ ); Figure S5. DSC curves (heating rate: $10{ }^{\circ} \mathrm{C} / \mathrm{min}$ ) of sucrose crystals produced from the $\mathrm{MeOH}$ extraction solution of $A$. gigas at a constant solvent volume of $3 \mathrm{~mL}$ under BMImTFSI anti-solvent composition variation conditions using the crystallization method; Figure S6. ${ }^{19} \mathrm{~F}-\mathrm{NMR}$ spectrum of $\mathrm{BMImPF}_{6}$ (solvent: $\mathrm{D}_{2} \mathrm{O}$ ); Figure S7. ${ }^{19} \mathrm{~F}-\mathrm{NMR}$ spectrum of sucrose crystals precipitated from $\mathrm{BMImPF}_{6}$. (A. gigas extraction solution/ILs: $3 \mathrm{~mL} / 3 \mathrm{~mL}(1: 1 \mathrm{v} / \mathrm{v})$, solvent: $\left.\mathrm{D}_{2} \mathrm{O}\right)$.

Acknowledgments: This research was supported by the Basic Science Research Program through the National Research Foundation of Korea (NRF) funded by the Ministry of Education (NRF-2016016008).

Author Contributions: Ji-Hun An and Alice Nguvoko Kiyonga performed the bulk of the experimental work. Woojin Yoon, Changjin Lim, and Minho Park performed some experiments. Kiwon Jung and Gyu-Hwan Park designed and supervised the work. Ji-Hun An, Alice Nguvoko Kiyonga, Minho Park, Younghwi Yun, Changjin Lim, and Kiwon Jung analyzed the data and wrote the manuscript.

Conflicts of Interest: The authors declare no conflict of interest.

\section{References}

1. Mullin, J.W. Crystallization; Lightning Source: La Vergne, TN, USA, 2001; Chapter 1; pp. 1-3.

2. Davey, R.; Garside, J. Form Molecules to Crystallizers an Introduction to Crystallization; Oxford University Press: Oxford, UK, 2000; Chapter 1; pp. 1-5.

3. Modi, S.R.; Dantuluri, A.K.R.; Puri, V.; Pawar, Y.B.; Nandekar, P.; Sangamwar, A.T.; Perumalla, S.R.; Sun, C.C.; Bansal, A.K. Impact of Crystal Habit on Biopharmaceutical Performance of Celecoxib. Cryst. Growth Des. 2013, 13, 2824-2832. [CrossRef]

4. Thakur, A.; Thipparaboina, R.; Kumar, D.; Gouthami, K.S.; Shastri, N.R. Crystal engineered albendazole with improved dissolution and material attributes. CrystEngComm 2016, 18, 1489-1494. [CrossRef]

5. Cashell, C.; Corcoran, D.; Hodnett, B.K. Effect of Amino Acid Additives on the Crystallization of L-Glutamic Acid. Cryst. Growth Des. 2005, 5, 593-597. [CrossRef]

6. Rogers, R.D.; Seddon, K.R. Ionic Liquids-Solvents of the Future? Science 2003, 302, 792-793. [CrossRef] [PubMed]

7. Rogers, R.D. Reflections on ionic liquids. Nature 2007, 447, 917-918. [CrossRef] [PubMed]

8. Reichert, W.M.; Holbrey, J.D.; Vigour, K.B.; Morgan, T.D.; Broker, G.A.; Rogers, R.D. Approaches to crystallization from ionic liquids: Complex solvents-complex results, or, a strategy for controlled formation of new supramolecular architectures? Chem. Commun. 2006, 46, 4767-4779. [CrossRef]

9. Kunov-Kruse, A.J.; Weber, C.C.; Rogers, R.D.; Myerson, A.S. The A Priori Design and Selection of Ionic Liquids as Solvents for Active Pharmaceutical Ingredients. Chem. Eur. J. 2017, 23, 5498-5508. [CrossRef] [PubMed]

10. Smith, K.B.; Bridson, R.H.; Leeke, G.A. Solubilities of Pharmaceutical Compounds in Ionic Liquids. J. Chem. Eng. Data 2011, 56, 2039-2043. [CrossRef]

11. Resende de Azevedo, J.; Letourneau, J.J.; Espitalier, F.; Re, M.I. Solubility of a New Cardioactive Prototype Drug in Ionic Liquids. J. Chem. Eng. Data 2014, 59, 1766-1773. [CrossRef]

12. Weber, C.C.; Kunov-Kruse, A.J.; Rogers, R.D.; Myerson, A.S. Manipulation of ionic liquid anion-solute-antisolvent interactions for the purification of acetaminophen. Chem. Commun. 2015, 51, 4294-4297. [CrossRef] [PubMed]

13. Smith, K.B.; Bridson, R.H.; Leeke, G.A. Crystallisation control of paracetamol from ionic liquids. CrystEngComm 2014, 16, 10797-10803. [CrossRef] 
14. Weber, C.C.; Kulkarni, S.A.; Kunov-Kruse, A.J.; Rogers, R.D.; Myerson, A.S. The Use of Cooling Crystallization in an Ionic Liquid System for the Purification of Pharmaceuticals. Cryst. Growth Des. 2015, 15, 4946-4951. [CrossRef]

15. Karthika, S.; Radhakrishnan, T.K.; Kalaichelvi, P. The role of hydrogen bonding propensity in tuning the morphology of crystals obtained from imidazolium based ionic liquids. J. Cryst. Growth 2017, 463, 168-175. [CrossRef]

16. Resende de Azevedo, J.; Espitalier, F.; Letourneau, J.J.; Ré, M.I. Antisolvent crystallization of a cardiotonic drug in ionic liquids: Effect of mixing on the crystal properties. J. Cryst. Growth 2017, 472, 29-34. [CrossRef]

17. Pusey, M.L.; Paley, M.S.; Turner, M.B.; Rogers, R.D. Protein Crystallization Using Room Temperature Ionic Liquids. Cryst. Growth Des. 2007, 7, 787-793. [CrossRef]

18. Judge, R.A.; Takahashi, S.; Longenecker, K.L.; Fry, E.H.; Zapatero, C.A.; Chiu, M.L. The Effect of Ionic Liquids on Protein Crystallization and X-ray Diffraction Resolution. Cryst. Growth Des. 2009, 9, 3463-3469. [CrossRef]

19. Kennedy, D.E.; Drummond, C.J.; Peat, T.S.; Newman, J. Evaluating Protic Ionic Liquids as Protein Crystallization Additives. Cryst. Growth Des. 2011, 11, 1777-1785. [CrossRef]

20. Li, X.; Xu, X.; Feng, Y.D.J.; Ge, L.; Zhang, M. The crystallization of lysozyme in the system of ionic liquid [BMIm][BF4]-water. Cryst. Res. Technol. 2008, 43, 1062-1068. [CrossRef]

21. Zhao, Y.; Chen, Z.; Wang, H.; Wang, J. Crystallization Control of $\mathrm{CaCO}_{3}$ by Ionic Liquids in Aqueous Solution. Cryst. Growth Des. 2009, 9, 4984-4986. [CrossRef]

22. Stoimenovski, J.; MacFarlane, D.R.; Bica, K.; Rogers, R.D. Crystalline vs. Ionic Liquid Salt Forms of Active Pharmaceutical Ingredients: A position paper. Pharm. Res. 2010, 27, 521-526. [CrossRef] [PubMed]

23. An, J.-H.; Kim, J.-M.; Chang, S.-M.; Kim, W.-S. Application of Ionic Liquid to Polymorphic Design of Pharmaceutical Ingredients. Cryst. Growth Des. 2010, 10, 3044-3050. [CrossRef]

24. An, J.-H.; Kim, W.-S. Antisolvent Crystallization Using Ionic Liquids As Solvent and Antisolvent for Polymorphic Design of Active Pharmaceutical Ingredient. Cryst. Growth Des. 2013, 13, 31-39. [CrossRef]

25. An, J.-H.; Jin, F.; Kim, H.S.; Ryu, H.C.; Kim, J.S.; Kim, H.M.; Kim, K.H.; Kiyonga, A.N.; Jung, K. Investigation of the Polymorphic Transformation of the Active Pharmaceutical Ingredient Clopidogrel Bisulfate Using the Ionic Liquid AEImBF 4 . Cryst. Growth Des. 2016, 16, 1829-1836. [CrossRef]

26. An, J.H.; Jin, F.; Kim, H.S.; Ryu, H.C.; Kim, J.S.; Kim, H.M.; Kiyonga, A.N.; Min, D.S.; Youn, W.; Kim, K.H.; et al. Application of ionic liquid to polymorphic transformation of antiviral/HIV drug adefovir dipivoxil. Arch. Pharm. Res. 2016, 39, 646-659. [CrossRef] [PubMed]

27. Shimpi, M.R.; Velaga, S.P.; Shah, F.U.; Antzutkin, O.N. Pharmaceutical Crystal Engineering Using Ionic Liquid Anion-Solute Interactions. Cryst. Growth Des. 2017, 17, 1729-1734. [CrossRef]

28. Pedireddi, V.R.; Shimpi, M.R.; Yakhmi, J.V. Room-Temperature Ionic Liquids: For a Difference in the Supramolecular Synthesis. Macromol. Symp. 2006, 241, 83-87. [CrossRef]

29. Lapkin, A.A.; Piucinski, P.K.; Cutler, M. Comparative Assessment of Technologies for Extraction of Artemisinin. J. Nat. Prod. 2006, 69, 1653-1664. [CrossRef] [PubMed]

30. Ventura, S.P.M.; e Silva, F.A.; Quental, M.V.; Mondal, D.; Freire, M.G.; Coutinho, J.A.P. Ionic-Liquid-Mediated Extraction and Separation Processes for Bioactive Compounds: Past, Present, and Future Trends. Chem. Rev. 2017, 117, 6984-7052. [CrossRef] [PubMed]

31. Kim, S.; Oh, H.-K.; Kim, J.-Y.; Homg, J.-W.; Cho, S.-I. A Review of Pharmacological Effects of Angelica gigas, Angelica sinensis, Angelica acutiloba and their Bioactive Compounds. J. Korean Orient. Med. 2011, 32, 1-24.

32. Son, C.Y.; Baek, I.H.; Song, G.Y.; Kang, J.S.; Kwon, K.I. Pharmacological Effect of Decursin and Decursinol Angelate from Angelica gigas Nakai. Yakhak Hoeji. 2009, 53, 303-313.

33. Xu, H.; Park, J.H.; Kim, Y.K.; Park, N.I.; Lee, S.Y.; Park, S.U. Optimization of growth and pyranocoumarins production in hairy root culture of Angelica gigas Nakai. J. Med. Plant. Res. 2009, 3, 978-981.

34. Ferreira, L.; Vidal, M.M.; Geraldes, C.F.G.C.; Gil, M.H. Preparation and characterisation of gels based on sucrose modified with glycidyl methacrylate. Carb. Pol. 2000, 41, 15-24. [CrossRef]

35. Brown, B.G.M.; Levy, H.A. Further Refinement of the Structure of Sucrose Based on Neutron-Diffraction Data. Acta. Cryst. 1973, B29, 790-797. [CrossRef]

36. Aquilano, D.; Angela, M.F.; Rubbo, M. Growth morphology of polar crystals: A comparison between theory and experiment in sucrose. J. Crystal Growth 1983, 61, 369-376. [CrossRef] 
37. Woensdregt, C.F. Hartman-Perdok Theory: Influence of Crystal Structure and Crystalline Interface on Crystal Growth. Farafay Discuss. 1993, 95, 97-107. [CrossRef]

38. Sounart, T.L.; Liu, J.; Voigt, J.A.; Huo, M.; Spoerke, E.D.; Mckenzie, B. Secondary Nucleation and Growth of ZnO. J. Am. Chem. Soc. 2007, 129, 15786-15793. [CrossRef] [PubMed]

39. Wiemers-Meyer, S.; Winter, M.; Nowak, S. Mechanistic insights into lithium ion battery electrolyte degradation-A quantitative NMR study. Phys. Chem. Chem. Phys. 2016, 18, 26595-26601. [CrossRef] [PubMed]

40. Plakhotnyk, A.V.; Ernst, L.; Schmutzler, R. Hydrolysis in the system LiPF6—propylene carbonate-dimethyl carbonate- $\mathrm{H}_{2} \mathrm{O}$. J. Fluorine Chem. 2005, 126, 27-31. [CrossRef]

41. Campion, C.L.; Li, W.; Lucht, B.L. Thermal Decomposition of LiPF6-Based Electrolytes for Lithium-Ion Batteries. J. Electrochem. Soc. 2005, 152, A2324-A2337. [CrossRef]

(C) 2017 by the authors. Licensee MDPI, Basel, Switzerland. This article is an open access article distributed under the terms and conditions of the Creative Commons Attribution (CC BY) license (http:/ / creativecommons.org/licenses/by/4.0/). 